\title{
Associations Between Physical Activity and Perceived School Performance of Young Adolescents in Health Behavior in School-Aged Children Countries
}

\author{
Kwok W. Ng, Gorden Sudeck, Adilson Marques, Alberto Borraccino, Zuzana Boberova, \\ Jana Vasickova, Riki Tesler, Sami Kokko, and Oddrun Samdal
}

\begin{abstract}
Background: Regular physical activity and doing well in school are important for growing adolescents. In this study, the associations between physical activity and perceived school performance (PSP) are examined together. Methods: Young adolescents from 42 countries $(n=193,949)$ in Europe and Canada were examined for associations between self-reported moderate to vigorous physical activity (MVPA) and PSP. Multinominal analyses were conducted with 0 to 2 days of MVPA and below average PSP as reference categories. Adjusted odds ratios and 95\% confidence intervals were reported for pooled data and individual countries after controlling for family affluence scale. Results: Girls had better PSP than boys, yet more boys participated in daily MVPA than girls. The associations between PSP and MVPA were inverted $U$ shaped. The strongest association for very good PSP was among young adolescents who reported 5 to 6 days MVPA (odds ratios $=2.3 ; 95 \%$ confidence interval, 2.1-2.4) after controlling for family affluence scale. Conclusions: Young adolescents with average or better PSP took part in at least 3 days of MVPA in a week, suggesting that participating in some MVPA was positively associated with PSP. More days of MVPA in a week, especially for young adolescents with below average PSP, would be beneficial for health and school performance.
\end{abstract}

Keywords: transition, academic performance, sport, adolescence

The overall health benefits of physical activity (PA) are well documented. ${ }^{1}$ Among youth, PA is known to have positive effects on cardiovascular, bone, and metabolic health; improving fitness; weight status; sleep; and mental health. ${ }^{1,2}$ The current guidelines for children between 5 and 18 years old to achieve health-enhancing PA are to engage in moderate to vigorous PA (MVPA) for at least 60 minutes per day. ${ }^{3,4}$ Adherence to this guideline is remarkably low around the word. ${ }^{5}$ The decline of participation with increasing age is also dramatic, from $23.2 \%$ among 11 -year-olds to $14.0 \%$ among 15 year-olds. ${ }^{6}$ Perceived lack of time and involvement in other activities (eg, school homework) are commonly reported as personal barriers to young adolescents' PA participation. ${ }^{7}$ Young adolescents or early adolescence has been defined for children aged between 10 and 15 years. ${ }^{8}$ As adolescents get older and receive increased academic workloads, ${ }^{9}$ they may feel that they have less time to be physically active. In addition, more serious competition opportunities in sports

\footnotetext{
$\mathrm{Ng}$ is with the School of Educational Sciences and Psychology, University of Eastern Finland, Joensuu, Finland; and the Physical Activity for Health Research Cluster, Department of Physical Education and Sport Sciences, University of Limerick, Limerick, Ireland. Sudeck is with the Department of Education and Health Research, Institute of Sports Science, Eberhard Karls University of Tuebingen, Tuebingen, Germany. Marques is with CIPER, Faculty of Human Kinetics, University of Lisbon, Lisbon, Portugal. Borraccino is with the Department of Public Health and Paediatrics, University of Turin, Turin, Italy. Boberova is with the Institute of Biology and Ecology, Faculty of Science, Pavol Jozef Safarik University, Kosice, Slovakia. Vasickova is with the Department of Social Science in Kinanthropology, Faculty of Physical Culture, Palacky University, Olomouc, Czech Republic. Tesler is with the Department of Health Management, Ariel University, Ariel, Israel. Kokko is with the Faculty of Sport and Health Sciences, University of Jyväskylä, Jyväskylä, Finland. Samdal is with the Department of Health Promotion and Development, University of Bergen, Bergen, Norway. Ng (kwok.ng@ hbsc.org) is corresponding author.
}

arise as children get older, and these could inhibit time spent on carrying out schoolwork. ${ }^{10}$

There is a growing body of research that investigates the association between PA and short-term academic performance (eg, grades, test scores, grade repetition), as well as long-term educational attainment (eg, level of education, college enrolment), but with mixed findings. ${ }^{11-13}$ Less disputed is the importance for good health, the individual's perceptions of competence in scholastic and physical activities during young adolescence as part of their self-concept. ${ }^{14}$ A parabola trend has been suggested during early adolescence, whereby self-concept is considered to decline from the ages of 9 to 11 years and only begin to increase again after the age of 15 years. ${ }^{15,16}$ Therefore, more awareness of the perception of scholastic and PA levels of adolescents between these years can be useful for designing school-based interventions to increase both PA and school performance.

The optimal dose of PA in terms of type, frequency, duration, and intensity that is associated with educational benefits at different ages also remains unclear. Koivusilta et $\mathrm{al}^{17}$ reported negative longitudinal associations of daily PA participation with long-term educational attainment. They concluded that great amounts of time invested in physical activities had detrimental effects on coping with school demands, which in turn, may lead to lower academic performance. This may explain how some studies arrived at null findings ${ }^{18,19}$ or even negative effects of PA on educational attainment. ${ }^{20}$ There have also been positive conclusions on the beneficial effects of PA on educational outcomes, ${ }^{21}$ and variances in the conclusions have led researchers to call for further research that can confirm the scientific understanding in relationships between PA and educational outcomes. ${ }^{13,22,23}$ Furthermore, there appears to be differences between sexes; for example, researchers have reported educational impact for physically active girls but not for boys. ${ }^{24}$ 
As such, more research is necessary to establish knowledge on the circumstances that foster or diminish a positive relationship between PA and perceived educational outcomes.

Critics from systematic literature reviews on the relationships between PA and academic outcomes have often cited a lack of comparable measures and differences in the dose of PA, as well as insufficient controlling for potential confounders, like socioeconomic status (SES). ${ }^{23}$ Furthermore, most young adolescents experience transitions from primary- to secondary-level education between the ages of 11 and 15 years. Traditional measures of academic outcomes also vary between the school systems and countries. To consider these differences, self-reported measures of perceived academic performance through a standardized instrument allow for intercountry comparisons across young adolescents. ${ }^{25}$ Such measures require individuals to reflect upon their own performance. Perceived school performance (PSP) ${ }^{26}$ has been reported to be a good predictor of characteristics associated with academic performance, such as motivation to learn, approaches to learning, and resilience. ${ }^{27,28}$ Self-perceptions are commonly used in developmental psychology to investigate a multitude of constructs among young adolescents, such as self-esteem ${ }^{29}$ and identity. ${ }^{30}$ That is to say, young adolescents who may perceive to have good academic performance do so because they have confidence with themselves and can associate themselves with that identity, ${ }^{31}$ yet evidence of the associations with PA are lacking.

Given the need for more research to better understand the relationships between PA and academic performance across the transition of young adolescents, PSP can be used to examine the associations with PA between 11-, 13-, and 15-year-olds in 42 countries and regions in Europe and Canada. Previous research has determined that there are inconsistent findings in the associations, which may be due to changes in the frequency of PA. Therefore, the present analysis focuses on different "doses" of weekly MVPA and studies sex-specific associations of different PA doses across the different age cohorts. In addition, consistencies and differences across countries are explored.

\section{Methods}

\section{Procedures}

The data were based on the World Health Organization's collaborative, cross-national Health Behavior in School-Aged Children (HBSC) study across 48 countries and regions within Europe and North America. The HBSC study involves young adolescents who express their quantification of key health behaviors, health indicators, and contextual variables. ${ }^{32}$ The research covers various aspects of health and health behavior of young adolescents in social contexts (family, peers, and school). Data collection in each country followed a standardized protocol, including translation, sampling methods, and ethical practice.

Our primary data came from 42 countries participating in the 2013-14 HBSC survey. The specific population targeted for sampling included young adolescents aged 11, 13, and 15 years. Cluster sampling was used, with the primary sampling unit being the school class. The nationally representative data were obtained by each country based on a calculation of at least 1500 young adolescents from each year group, where possible (Greenland's young adolescent population was less than 1500). A total of 219,810 young adolescents completed the survey. The data were cleaned through a stepwise approach by removing missing cases of age $(n=1854$; $0.08 \%$ ), family affluence scale (FAS3; $\mathrm{n}=17,834 ; 8.1 \%$ ), MVPA $(\mathrm{n}=4217 ; 2.2 \%)$, and PSP $(\mathrm{n}=1956 ; 1.0 \%)$. Young adolescents completed questionnaires during ordinary class hours. Each country ensured approval by the ethics committee or relevant board (varied by country). Informed consent for the young adolescents' participation in the study was obtained from their parents or guardian. Participation in the study was fully voluntary and anonymous.

\section{Measures}

All questionnaire items were piloted with HBSC countries. The standard English version of the international questionnaire was translated for the specific national language(s) and cultural contexts of the participating countries.

Perceived School Performance. The HBSC study was conducted in countries with different school and grading systems. A single item was used across all the schools in the study, with the purpose of gauging school performance in the following way: "In your opinion, what does your class teacher(s) think about your school performance compared to your classmates?" Respondents can choose between the following response options: "My teacher (s) think(s) my school performance is . . . 1 = very good; $2=$ good; 3 = average; and $4=$ below average." This PSP question has been used with the same wording and response format in the HBSC study since 1986. A validation study for this single item was performed by Felder-Puig et al. ${ }^{25}$ Correlations between .51 and .65 , representing large effect sizes, were reported between school grades and school performance through this particular item ${ }^{25}$; moreover, the item had strong test-retest reliability scores. ${ }^{33,34}$ In addition, the mandatory HBSC survey consists of over 40 questions, and when compared with other items in the survey, the PSP item was the strongest correlated item to actual academic grades. The response data were based on reversed scoring, with a higher score representing higher PSP. Perceptions of "below average" were set as a reference category for analyses because we expect there to be improvements in the associations as PSP increases.

Moderate to Vigorous PA. A self-reported item on PA over the previous 7 days was used. MVPA was written with some definitions of PA with some country-specific examples of where it can take place. The individuals were then asked, "Over the past 7 days, on how many days were you physically active for a total of at least 60 minutes per day? Please add up all the time you spent in physical activity each day." The response category range was between 0 and 7 days. This item has been reported to have reasonable validity ${ }^{35}$ and moderate reliability, ${ }^{36}$ thus its suitability for surveillance data. ${ }^{37}$ The number of categories were reduced by half, from 8 categories to $4(0-2 \mathrm{~d}, 3-4 \mathrm{~d}, 5-6 \mathrm{~d}$, and $7 \mathrm{~d})$. The reliability of a 4-category selection of this PA variable has moderate agreement. ${ }^{36}$ It was important to include the last category because respondents who report 7 days are considered as meeting the internationally adopted PA guidelines for health. ${ }^{3,4}$

Demographic Covariates. Individuals reported their sex as boy or girl and reported the month and year they were born. This latter information, combined with the date of survey completion, was used to create an estimate of age. Age categories were created from age ranges that included 10.5 to 12.5 years $(11 \mathrm{y}), 12.51$ to 14.5 years $(13 \mathrm{y})$, and 14.51 to 16.50 years $(15 \mathrm{y})$. Estimated ages outside of the 10.5- to 16.5-year range were removed from the analyses. Social inequalities were determined by the FAS3. ${ }^{38}$ FAS3 is a child-friendly proxy measure of family wealth, consisting of 6 material possessions accessible to the young adolescents. Individuals reported how many cars the family owned, how 
many computers their family owned, the number of bathrooms in their home, the number of times the family had travelled out of their country for a holiday in the previous year, whether they had their own room, and whether they had a dishwasher at home. The response options varied depending on the question, with a score range from 0 to 13 . The Rankit formula, ranked 0 to 1 , was applied at each country level to express the social inequalities within each country.

\section{Statistical Analyses}

Descriptive statistics with the chi-square test of independence were applied between sexes in the categories of PSP. The differences between boys and girls were statistically significant, and therefore, analyses were conducted separately for boys and girls. In addition, chi-square tests of independence were conducted for boys and girls to analyze the transition in age cohorts in the proportion of daily MVPA. Risk ratios (RR) were calculated to report the effect sizes for sex differences in PSP and MVPA.

Multilevel analyses were carried out at the class level for PSP, with students at level 1 and classes at level 2. ${ }^{39}$ These analyses were based on the assumption that the independence of the pupils' PSP was compromised owing to class effects. To test the assumption that the clustering was affected by the class level, the intraclass correlational coefficient (ICC) was computed for single (ICC1) and average (ICC2) scores. ${ }^{40,41}$ Overall sample and individual country test results (Supplementary Table 1 [available online]) were insufficiently independent with ICC1 $(\rho<.0001)$ and ICC2 $(\rho=.5550)$; thus, the evidence did not warrant or justify executing the complexity of between, within, and mixed-level models. That is to say that an ICC1 value indicated that less than $1 \%$ of the observed variance in pupils' PSP was due to systematic between-classroom differences compared with the total variance in achievement scores. In addition, the value of ICC2 represented $55 \%$ of the observed total variance in the classroom average scores occurring at the classroom level. Therefore, analyses were performed at a single level with multinominal logistic regression analyses.

Perceived school performance was entered as the dependent variable, with the below average grade as the reference category. FAS3 and sex were included in a null model. In the full model, categories of MVPA were entered with 0 to 2 days as the reference category. The multinominal logistic regression reduces the error from repeated comparisons between 2 categorical variables. Adjusted odds ratios (ORs) were reported with $95 \%$ confidence intervals (CIs). The logistic regression model for the entire sample was applied and then repeated separately for each country. All analyses were computed using IBM Statistical Package for the Social Sciences (version 24.0; IBM Corp, Chicago, IL).

\section{Descriptive Results}

After cleaning the data file for missing and spurious cases, a total of 193,949 young adolescents from 42 countries reported their daily MVPA habits and PSP. There were slightly more girls $(\mathrm{n}=$ $99,851 ; 51.5 \%)$ than boys $(\mathrm{n}=94,098 ; 48.5 \%)$. There were fewer 11 -year- olds $(n=61,241 ; 31.6 \%)$ than $13-(n=67,463 ; 34.8 \%)$ and 15 -year-olds $(n=65,245 ; 33.6 \%)$, and the overall distribution of FAS3 was $19.7 \%$ for low, $60.5 \%$ for medium, and $19.8 \%$ for high groups. The country with the most number of respondents was Canada $(\mathrm{n}=11,220 ; 5.8 \%)$, and the least was from Greenland $(\mathrm{n}=709 ; 0.37 \%)$, with the expected mean $(2.38 \%)$ representation of samples at the country level being equal to the actual mean $(2.38 \%)$.

There were significantly more boys $(5.4 \%)$ with below average PSP than girls $(3.7 \%$; RR $=1.48 ; 95 \% \mathrm{CI}, 1.42-1.55)$, whereas more girls $(26.0 \%)$ than boys $(21.7 \%)$ reported excellent PSP $(\mathrm{RR}=1.20 ; 95 \% \mathrm{CI}, 1.18-1.22)$. More girls reported low or no levels of MVPA $(0-2 \mathrm{~d}$; RR $=1.44 ; 95 \% \mathrm{CI}, 1.41-1.46 ; 0-4 \mathrm{~d}$; $\mathrm{RR}=1.27$; 95\% CI, 1.26-1.28) than boys. However, more boys reported daily MVPA than girls ( $\mathrm{RR}=1.61 ; 95 \% \mathrm{CI}, 1.58-1.64)$.

Across all countries, $24 \%$ perceived their school performance as very good, $43 \%$ as good, $28 \%$ as average, and $5 \%$ as below average (see Supplementary Table 2 [available online]). The estimates for below average were the least in Albania (boys $=2.5 \%$; girls $=1 \%$ ) and the highest in France (boys $=11.4 \%$; girls $=8.8 \%$ ). In addition, FAS3 was positively associated with higher PSP and controlled for in a multinominal logistic regression analyses. In the higher age groups, the perception of one's own school performance turned out to be increasingly worse. The proportion with a good or very good PSP decreased from $77 \%$ for 11 -year-olds to $65 \%$ for 13 -year-olds and $60 \%$ for 15 year-olds. The proportion of boys who reported their PSP as below average and average was much higher than for girls, and conversely, more girls than boys reported their PSP as good or very good (Table 1). Statistically significant sex differences were found in 31 countries and regions, whereby a higher percentages of girls reported good or very good PSP $(P<.05)$. Only in Portugal was there a greater proportion of boys reporting good or very good PSP.

For girls, the same pattern of MVPA was observed in 40 countries, with PA lessening along with increasing age (see Table 2 and Supplementary Table 3 [available online]; there were only 2 exceptions with nonsignificant age differences in Italy and the Netherlands). For boys, there were also significant age differences in 32 countries, with the lowest proportion of boys who reported daily MVPA at age 15 . For a further 8 countries, however, no significant age differences were found, and in 2 countries, the Republic of Moldova and Norway, the lowest proportion was among 13-year-old pupils.

\section{Main Results}

After pooling all the data, the results of the multinominal logistic regression analyses revealed that the associations between the number of days with MVPA and odds for improved PSP followed inverted $U$ patterns for both girls and boys after controlling for FAS3 (Figure 1). Regardless of sex and age group, across almost all PSP categories, compared with below average PSP (as PSP reference group), a frequency of 5 to 6 days of MVPA exhibited the highest OR for better PSP. Only the comparison of average with below average PSP among 11-year-old girls and boys did not follow this pattern. There were also significant positive associations with good and very good PSP for boys and girls who reported taking part in daily MVPA. The highest OR based on the frequency categories of MVPA was 5 to 6 days MVPA and increased as the PSP improved. Furthermore, the CI of the ORs crossed 1.0 among girls and boys of all age groups in the average PSP group and reported daily MVPA, indicating nonsignificant associations between average PSP and daily MVPA when compared with below average PSP and 0 to 2 days MVPA.

Although quite comparable patterns can be described overall (with respect to highest OR for 5-6 d MVPA), the strength of the associations differed across age groups and between boys and girls. 
Table 1 Gender Difference Among Proportion (\%) of PSP From the 2013-14 HBSC Study

\begin{tabular}{|c|c|c|c|c|c|c|c|c|c|c|c|}
\hline & \multicolumn{5}{|c|}{ Boys } & \multicolumn{5}{|c|}{ Girls } & \multirow{2}{*}{$\chi^{2}$} \\
\hline & $\begin{array}{c}\text { Below } \\
\text { average }\end{array}$ & Average & Good & $\begin{array}{l}\text { Very } \\
\text { good }\end{array}$ & $\begin{array}{c}\text { Total } \\
\text { (n) }\end{array}$ & $\begin{array}{c}\text { Below } \\
\text { average }\end{array}$ & Average & Good & $\begin{array}{l}\text { Very } \\
\text { good }\end{array}$ & $\begin{array}{c}\text { Total } \\
\text { (n) }\end{array}$ & \\
\hline Total country & 5.40 & 29.90 & 42.90 & 21.70 & 94,098 & 3.70 & 26.90 & 43.50 & 26.00 & 99,851 & $<.001$ \\
\hline Albania & 2.50 & 17.80 & 31.40 & 48.30 & 2228 & 1.00 & 11.30 & 21.10 & 66.50 & 2403 & $<.001$ \\
\hline Armenia & 4.10 & 29.00 & 42.60 & 24.30 & 1398 & 1.50 & 21.70 & 50.20 & 26.50 & 1625 & $<.001$ \\
\hline Austria & 4.80 & 33.00 & 42.50 & 19.60 & 1444 & 4.00 & 32.50 & 43.90 & 19.50 & 1657 & .67 \\
\hline Belgium (Flemish) & 8.90 & 34.20 & 38.80 & 18.10 & 2129 & 5.30 & 31.40 & 44.60 & 18.70 & 1816 & $<.001$ \\
\hline Belgium (French) & 8.80 & 37.70 & 39.20 & 14.30 & 2636 & 8.90 & 39.10 & 38.80 & 13.20 & 2750 & .60 \\
\hline Bulgaria & 4.40 & 17.50 & 45.90 & 32.30 & 2219 & 2.00 & 14.90 & 43.70 & 39.40 & 2065 & $<.001$ \\
\hline Canada & 5.50 & 27.80 & 45.60 & 21.00 & 5447 & 3.70 & 22.00 & 45.00 & 29.30 & 5773 & $<.001$ \\
\hline Croatia & 4.10 & 21.00 & 35.60 & 39.40 & 2333 & 2.70 & 17.10 & 27.00 & 53.10 & 2404 & $<.001$ \\
\hline Czech Republic & 8.00 & 39.50 & 39.30 & 13.20 & 2287 & 5.50 & 36.00 & 41.60 & 16.90 & 2522 & $<.001$ \\
\hline Denmark & 3.70 & 28.20 & 49.60 & 18.50 & 1614 & 3.50 & 27.60 & 48.60 & 20.40 & 1876 & .56 \\
\hline England & 3.50 & 25.20 & 48.10 & 23.20 & 2177 & 2.00 & 17.50 & 50.40 & 30.00 & 2154 & $<.001$ \\
\hline Estonia & 4.40 & 38.10 & 42.30 & 15.20 & 1962 & 3.50 & 29.50 & 48.30 & 18.70 & 1964 & $<.001$ \\
\hline Finland & 4.90 & 32.50 & 45.40 & 17.20 & 2696 & 2.60 & 24.00 & 48.80 & 24.60 & 2876 & $<.001$ \\
\hline France & 11.40 & 35.30 & 37.00 & 16.30 & 2462 & 8.80 & 33.80 & 40.10 & 17.30 & 2461 & .01 \\
\hline Germany & 6.70 & 45.10 & 39.00 & 9.20 & 2779 & 5.20 & 41.70 & 42.80 & 10.20 & 2686 & .002 \\
\hline Greece & 3.90 & 22.10 & 44.20 & 29.90 & 2000 & 2.00 & 18.90 & 39.90 & 39.20 & 2018 & $<.001$ \\
\hline Greenland & 4.60 & 26.70 & 49.00 & 19.70 & 345 & 4.70 & 29.10 & 46.40 & 19.80 & 364 & .89 \\
\hline Hungary & 6.60 & 42.70 & 35.10 & 15.50 & 1808 & 4.80 & 44.00 & 34.10 & 17.10 & 1853 & .06 \\
\hline Iceland & 5.40 & 22.80 & 43.90 & 27.90 & 4630 & 3.40 & 18.90 & 41.80 & 35.90 & 4765 & $<.001$ \\
\hline Ireland & 5.00 & 24.40 & 45.20 & 25.30 & 1346 & 2.50 & 20.70 & 43.40 & 33.30 & 2163 & $<.001$ \\
\hline Israel & 5.40 & 19.90 & 36.00 & 38.70 & 2391 & 3.80 & 19.00 & 36.80 & 40.30 & 2730 & .04 \\
\hline Italy & 10.00 & 37.90 & 37.50 & 14.50 & 1915 & 6.00 & 34.40 & 39.50 & 20.10 & 1923 & $<.001$ \\
\hline Latvia & 6.90 & 40.80 & 42.00 & 10.20 & 2521 & 3.60 & 40.30 & 42.90 & 13.20 & 2836 & $<.001$ \\
\hline Lithuania & 5.30 & 38.40 & 39.00 & 17.30 & 2718 & 3.00 & 33.40 & 43.60 & 20.00 & 2734 & $<.001$ \\
\hline Luxembourg & 4.70 & 31.90 & 44.50 & 18.80 & 1244 & 4.50 & 29.30 & 47.10 & 19.00 & 1428 & .46 \\
\hline Malta & 3.70 & 21.00 & 44.40 & 30.80 & 999 & 3.30 & 21.80 & 41.70 & 33.30 & 1013 & .52 \\
\hline MKD & 1.60 & 9.30 & 33.70 & 55.40 & 1972 & 1.40 & 7.30 & 26.60 & 64.70 & 1994 & $<.001$ \\
\hline Republic of Moldova & 4.00 & 27.70 & 48.60 & 19.80 & 2275 & 2.40 & 21.40 & 53.10 & 23.10 & 2266 & $<.001$ \\
\hline The Netherlands & 4.60 & 29.10 & 49.30 & 17.00 & 1885 & 3.50 & 27.80 & 52.40 & 16.30 & 2004 & .15 \\
\hline Norway & 4.00 & 26.60 & 47.00 & 22.40 & 1349 & 3.30 & 24.10 & 45.60 & 27.10 & 1503 & .02 \\
\hline Poland & 6.20 & 37.10 & 41.00 & 15.80 & 2070 & 3.50 & 34.40 & 42.30 & 19.70 & 2149 & $<.001$ \\
\hline Portugal & 5.60 & 36.70 & 41.80 & 15.90 & 2067 & 4.80 & 44.70 & 39.30 & 11.30 & 2298 & $<.001$ \\
\hline Romania & 3.40 & 29.80 & 48.20 & 18.60 & 1595 & 3.00 & 26.90 & 49.10 & 21.00 & 1878 & .14 \\
\hline Russian Federation & 4.50 & 39.60 & 42.50 & 13.40 & 1804 & 4.20 & 33.90 & 46.10 & 15.80 & 2380 & .001 \\
\hline Scotland & 6.80 & 30.60 & 44.70 & 17.90 & 2639 & 4.40 & 25.90 & 44.00 & 25.80 & 2733 & $<.001$ \\
\hline Slovakia & 3.90 & 24.90 & 47.30 & 23.90 & 2498 & 3.00 & 19.00 & 49.80 & 28.20 & 2628 & $<.001$ \\
\hline Slovenia & 4.60 & 21.00 & 47.20 & 27.10 & 2267 & 2.50 & 19.50 & 48.80 & 29.10 & 2400 & $<.001$ \\
\hline Spain & 7.40 & 28.40 & 45.10 & 19.10 & 3140 & 4.30 & 27.70 & 44.00 & 24.00 & 3468 & $<.001$ \\
\hline Sweden & 4.40 & 34.30 & 45.70 & 15.50 & 3381 & 3.00 & 34.10 & 48.80 & 14.10 & 3523 & .001 \\
\hline Switzerland & 4.40 & 26.40 & 52.00 & 17.30 & 2986 & 3.40 & 24.90 & 53.00 & 18.70 & 3082 & .09 \\
\hline Ukraine & 5.80 & 40.80 & 35.30 & 18.10 & 1979 & 2.30 & 35.40 & 44.00 & 18.40 & 2290 & $<.001$ \\
\hline Wales & 3.60 & 22.10 & 51.50 & 22.80 & 2463 & 2.00 & 17.20 & 46.90 & 33.90 & 2396 & $<.001$ \\
\hline
\end{tabular}

Abbreviations: HBSC, Health Behavior in School-Aged Children; MKD, Former Yugoslavian Republic of Macedonia; PSP, perceived school performance.

The strongest associations were among 13-year-old girls $(\mathrm{OR}=$ 3.05 ; 95\% CI, 2.56-3.63), whereas the weakest associations were among 15 -year-old boys $(\mathrm{OR}=1.62 ; 95 \% \mathrm{CI}, 1.41-1.86)$ and girls $(\mathrm{OR}=2.12 ; 95 \%$ CI, 1.81-2.48). The positive associations between all groups in comparison with the reference categories increased as PSP rose.

There were many similarities in the associations across individual countries, although some distinct country differences 

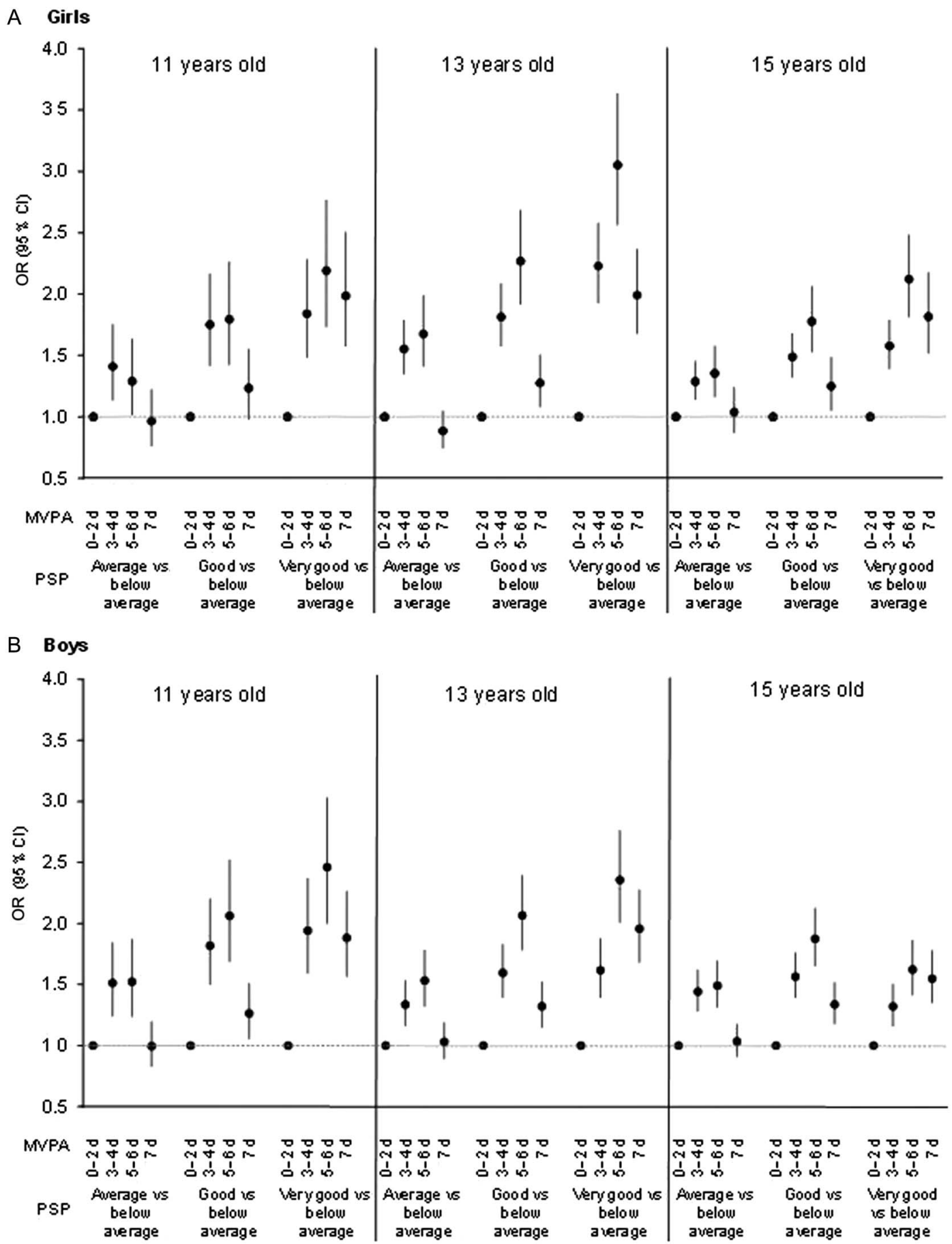

Figure 1 - Multinomial age-specific OR and 95\% CI for PSP on weekly number of days with at least 60 min of MVPA in girls (A) and boys (B). Pooled data of 42 countries, 2013-14 HBSC study. PSP (below the average, average, good, and very good [reference category: below average]); MVPA (0-2, 3-4, 5-6, and $7 \mathrm{~d}$ with at least $60 \mathrm{~min}$ of MVPA [reference category: 0-2 d]). CI indicates confidence interval; HBSC, Health Behavior in School-Aged Children; MVPA, moderate to vigorous physical activity; OR, odds ratio; PSP, perceived school performance.

exist (Table 3). There were 30 (3-4 d), 33 (5-6 d), and 21 (7 d) countries where the association with very good PSP was statistically significant compared with individuals with below average PSP. Specifically, young adolescents in Estonia, who reported daily MVPA, were 1.8 times more likely to report average PSP than below average PSP and 0 to 2 days of MVPA.
In France $(\mathrm{OR}=0.69 ; 95 \% \mathrm{CI}, 0.50-0.94)$ and Slovenia $(\mathrm{OR}=$ 0.56 ; $95 \% \mathrm{CI}, 0.35-0.89$ ), there were negative associations. There were 10 countries with an association for good PSP and daily MVPA, and 2 countries (Slovenia and Wales) with negative associations. Half of the countries $(n=21)$ had the same positive relationships for very good PSP with daily MVPA, and none reported negative associations. 
Table 2 Changes (Chi-Square) in Proportion of Young Adolescents Who Took Part in Daily MVPA, Stratified by Gender From the 2013-14 HBSC Study

\begin{tabular}{|c|c|c|c|c|c|c|c|c|c|c|c|c|c|c|}
\hline & \multicolumn{7}{|c|}{ Boy } & \multicolumn{7}{|c|}{ Girl } \\
\hline & \multicolumn{2}{|c|}{$11 \mathrm{y}$} & \multicolumn{2}{|c|}{$13 y$} & \multicolumn{2}{|c|}{$15 \mathrm{y}$} & \multirow[b]{2}{*}{$\chi^{2}$} & \multicolumn{2}{|c|}{$11 \mathrm{y}$} & \multicolumn{2}{|c|}{$13 y$} & \multicolumn{2}{|c|}{$15 y$} & \multirow[b]{2}{*}{$\chi^{2}$} \\
\hline & Count & MVPA & Count & MVPA & Count & $\overline{\text { MVPA }}$ & & Count & MVPA & Count & $\overline{\text { MVPA }}$ & Count & MVPA & \\
\hline & $\mathbf{n}$ & $\%$ & $\mathbf{n}$ & $\%$ & $\mathbf{n}$ & $\%$ & $P$ & $\mathbf{n}$ & $\%$ & $\mathbf{n}$ & $\%$ & $\mathbf{n}$ & $\%$ & $P$ \\
\hline Total country & 29,737 & 29.7 & 32,979 & 24.7 & 31,382 & 20.7 & $<.001$ & 31,504 & 20.8 & 34,484 & 15.2 & 33,863 & 10.8 & $<.001$ \\
\hline Albania & 739 & 37.5 & 731 & 38 & 758 & 28.8 & $<.001$ & 759 & 30.7 & 806 & 19.9 & 838 & 14 & $<.001$ \\
\hline Armenia & 587 & 29.1 & 487 & 29.8 & 324 & 25 & .30 & 614 & 20.2 & 502 & 19.5 & 509 & 14.3 & .03 \\
\hline Austria & 484 & 33.1 & 479 & 28.8 & 481 & 17.9 & $<.001$ & 503 & 26.2 & 484 & 13 & 670 & 5.5 & $<.001$ \\
\hline $\begin{array}{l}\text { Belgium } \\
\text { (Flemish) }\end{array}$ & 655 & 20.9 & 553 & 17.9 & 921 & 16.5 & .08 & 675 & 14.2 & 474 & 12.2 & 667 & 6.7 & $<.001$ \\
\hline $\begin{array}{l}\text { Belgium } \\
\text { (French) }\end{array}$ & 910 & 28.6 & 894 & 20.9 & 832 & 15.4 & $<.001$ & 898 & 16.4 & 894 & 12.2 & 958 & 11 & $<.001$ \\
\hline Bulgaria & 656 & 41.9 & 727 & 34.7 & 836 & 26.3 & $<.001$ & 713 & 30.2 & 664 & 20.2 & 688 & 18 & $<.001$ \\
\hline Canada & 1281 & 36.2 & 2074 & 31.7 & 2092 & 27.2 & $<.001$ & 1423 & 27.3 & 2160 & 19.3 & 2190 & 13.8 & $<.001$ \\
\hline Croatia & 676 & 38.8 & 805 & 32.5 & 852 & 24.2 & $<.001$ & 772 & 26.4 & 847 & 19 & 785 & 11.8 & $<.001$ \\
\hline Czech Republic & 697 & 29 & 765 & 27.2 & 825 & 19.5 & $<.001$ & 785 & 22.9 & 852 & 20.5 & 885 & 12.8 & $<.001$ \\
\hline Denmark & 485 & 17.3 & 582 & 16.3 & 547 & 15.7 & .79 & 546 & 11.4 & 675 & 11 & 655 & 7.5 & .04 \\
\hline England & 822 & 22.7 & 657 & 22.8 & 698 & 17.6 & .02 & 722 & 20.6 & 736 & 13.2 & 696 & 9.6 & $<.001$ \\
\hline Estonia & 626 & 21.2 & 711 & 22.5 & 625 & 18.7 & .23 & 659 & 15 & 679 & 11.8 & 626 & 9.3 & .01 \\
\hline Finland & 904 & 46.6 & 877 & 29.3 & 915 & 21.7 & $<.001$ & 970 & 33.6 & 922 & 22.9 & 984 & 13.1 & $<.001$ \\
\hline France & 705 & 23.8 & 972 & 15.7 & 785 & 13.2 & $<.001$ & 709 & 10.3 & 962 & 8.5 & 790 & 5.9 & .01 \\
\hline Germany & 774 & 24.4 & 993 & 17.2 & 1012 & 15.8 & $<.001$ & 762 & 15.1 & 924 & 12.1 & 1000 & 8.4 & $<.001$ \\
\hline Greece & 662 & 19.8 & 713 & 18.2 & 625 & 15.4 & .11 & 652 & 11.3 & 692 & 8.4 & 674 & 6.4 & .01 \\
\hline Greenland & 114 & 23.7 & 112 & 29.5 & 119 & 14.3 & .02 & 103 & 17.5 & 122 & 12.3 & 139 & 6.5 & .03 \\
\hline Hungary & 640 & 33.6 & 653 & 25.4 & 515 & 24.1 & $<.001$ & 675 & 23.9 & 631 & 14.3 & 547 & 11.2 & $<.001$ \\
\hline Iceland & 1469 & 31.2 & 1702 & 25.7 & 1459 & 24.2 & $<.001$ & 1528 & 21.5 & 1727 & 17.1 & 1510 & 13.9 & $<.001$ \\
\hline Ireland & 360 & 45 & 494 & 37 & 492 & 24.8 & $<.001$ & 562 & 31.5 & 782 & 15.9 & 819 & 9.4 & $<.001$ \\
\hline Israel & 873 & 22.9 & 799 & 14.4 & 719 & 10.4 & $<.001$ & 994 & 12.3 & 835 & 8.7 & 901 & 5.4 & $<.001$ \\
\hline Italy & 626 & 16.8 & 671 & 14.5 & 618 & 11.2 & .02 & 643 & 7.9 & 680 & 6.2 & 600 & 4.8 & .08 \\
\hline Latvia & 835 & 25 & 923 & 19.9 & 763 & 20.6 & .02 & 944 & 18.4 & 963 & 14 & 929 & 13.7 & .01 \\
\hline Lithuania & 927 & 27.3 & 926 & 28.3 & 865 & 23.2 & .04 & 973 & 20 & 985 & 12.9 & 776 & 11.5 & $<.001$ \\
\hline Luxembourg & 360 & 33.1 & 443 & 35.9 & 441 & 24.7 & .001 & 391 & 21 & 509 & 16.9 & 528 & 8.7 & $<.001$ \\
\hline Malta & 371 & 28.3 & 359 & 18.1 & 269 & 16.4 & $<.001$ & 347 & 20.7 & 367 & 11.7 & 299 & 8.7 & $<.001$ \\
\hline MKD & 655 & 36.9 & 611 & 31.8 & 706 & 27.2 & .001 & 660 & 29.8 & 647 & 24.4 & 687 & 11.8 & $<.001$ \\
\hline $\begin{array}{l}\text { Republic of } \\
\text { Moldova }\end{array}$ & 761 & 35.3 & 765 & 20.7 & 749 & 25 & $<.001$ & 755 & 29.3 & 738 & 24.7 & 773 & 22.1 & .01 \\
\hline The Netherlands & 614 & 23.8 & 654 & 20 & 617 & 21.2 & .26 & 638 & 14.9 & 693 & 16.6 & 673 & 12.6 & .12 \\
\hline Norway & 538 & 31.8 & 415 & 16.4 & 396 & 22.7 & $<.001$ & 580 & 19.3 & 471 & 12.1 & 452 & 7.3 & $<.001$ \\
\hline Poland & 725 & 35.3 & 702 & 29.6 & 643 & 25.2 & $<.001$ & 684 & 26.9 & 714 & 18.6 & 751 & 11.2 & $<.001$ \\
\hline Portugal & 613 & 25.6 & 863 & 24.3 & 591 & 17.6 & .002 & 736 & 14.5 & 882 & 5.9 & 680 & 5.1 & $<.001$ \\
\hline Romania & 484 & 39.3 & 549 & 27.1 & 562 & 20.8 & $<.001$ & 542 & 24 & 571 & 16.5 & 765 & 10.8 & $<.001$ \\
\hline $\begin{array}{l}\text { Russian } \\
\text { Federation }\end{array}$ & 501 & 24.6 & 711 & 21.1 & 592 & 20.6 & .23 & 731 & 18.2 & 919 & 14.7 & 730 & 10.8 & $<.001$ \\
\hline Scotland & 822 & 28.7 & 945 & 18.1 & 872 & 15.4 & $<.001$ & 873 & 20.4 & 974 & 11.8 & 886 & 10.5 & $<.001$ \\
\hline Slovakia & 714 & 38 & 980 & 30.7 & 804 & 26 & $<.001$ & 768 & 25.8 & 1102 & 20.2 & 758 & 13.2 & $<.001$ \\
\hline Slovenia & 755 & 27.7 & 809 & 24.4 & 703 & 21.5 & .02 & 752 & 17.8 & 828 & 13.5 & 820 & 7.4 & $<.001$ \\
\hline Spain & 812 & 39.3 & 1259 & 35 & 1069 & 28.5 & $<.001$ & 810 & 26.3 & 1367 & 19 & 1291 & 11.9 & $<.001$ \\
\hline Sweden & 1124 & 21.3 & 1018 & 14.1 & 1239 & 14.6 & $<.001$ & 1148 & 13.2 & 1033 & 10.6 & 1342 & 10.1 & .03 \\
\hline Switzerland & 884 & 25.8 & 1060 & 17.7 & 1042 & 12.5 & $<.001$ & 904 & 16.7 & 1124 & 9.7 & 1054 & 7.4 & $<.001$ \\
\hline Ukraine & 642 & 32.6 & 615 & 31.9 & 722 & 25.2 & .004 & 735 & 28.3 & 695 & 24.6 & 860 & 16.4 & $<.001$ \\
\hline Wales & 855 & 24 & 921 & 19.8 & 687 & 16.7 & .002 & 866 & 16.3 & 852 & 11.2 & 678 & 7.2 & $<.001$ \\
\hline
\end{tabular}

Abbreviations: HBSC, Health Behavior in School-Aged Children; MKD, Former Yugoslav Republic of Macedonia; MVPA, at least 60 min of moderate to vigorous physical activity daily. 


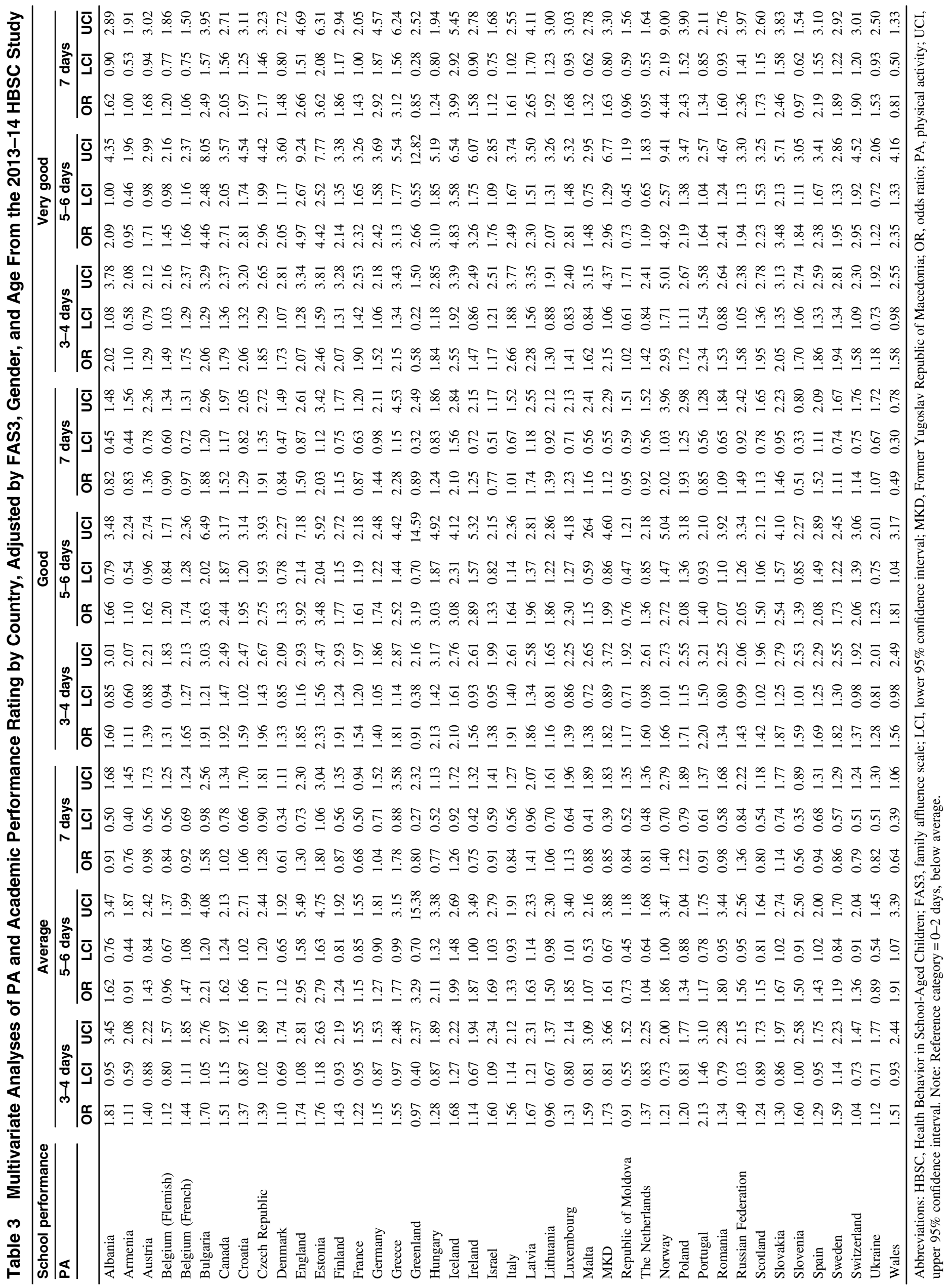




\section{Discussion}

In this study there were positive associations between PSP and MVPA levels. The findings were from an international study composed of 42 countries employing the same standardized instruments. The measures included a uniform way of social economic status through FAS3, as a confounder across different countries and applicable for young adolescents to complete ${ }^{42}$ and standardized measures of determining age. As young adolescents transitioned from the age of 11 to 15 years, fewer pupils reported very good PSP, and fewer took part in daily MVPA. This transition period during early adolescence is a critical one and affects both PSP and MVPA levels. ${ }^{43}$

The most striking finding was that the relationship between MVPA and PSP was not linear, but rather exhibited an inverted $U$ shape and was consistently reported across most countries. This was the case for all PSP categories that differed from below average PSP for both boys and girls. The probability for better PSP was the highest among reports of 5 to 6 days of MVPA. However, there were some subtle differences whereby 13-year-old girls who had very good PSP were over 3 times more likely to have reported 5 to 6 days of MVPA, whereas, for 13-year-old boys with very good PSP, they were 2.4 times more likely when compared with their same age and sex peers with 0 to 2 days of MVPA. Moreover, the strength of associations decreased as the age cohorts increased from 11 to 15 years among boys. These differences may be because boys have lower PSP and higher MVPA than girls. Young adolescent girls typically report higher PSP than boys ${ }^{44}$ and the results in this study mirror this. Kalman et $\mathrm{al}^{45}$ observed more boys who took part in daily MVPA than girls in the HBSC study between 2001 and 2010, and this pattern continued into the 2013-14 HBSC data, as also reported by Guthold et al. ${ }^{5}$ There were few countries with individual differences from the pooled results (Armenia, Germany, Greece, Latvia, Malta, Poland, Russian Federation, and Ukraine). Most of these countries are located in Eastern Europe. This could be an indication of specific coupling of the school and sports system, which, for example, makes higher activity volumes more compatible with school requirements. However, these are only initial observations and presumptions that should be followed up in further research, considering structural differences in the school system.

For boys and girls, as well as for all age groups, students who reported daily MVPA were not any more likely to have average PSP over below average PSP. This was a distinct finding, as all other frequencies of MVPA (between 3 and $6 \mathrm{~d}$ ) were positively associated with average PSP over below average PSP. This was observed in 22 countries. Although the international guidelines for health-enhancing PA for adolescents are daily MVPA for at least 60 minutes, ${ }^{3,4}$ students who already had low perceptions of their academic performance may need specific programs to elevate PSP and maintain PA levels. To take part in daily MVPA for at least 60 minutes, students must participate in PA outside of schooltime, and if the student already had below average PSP, they may not be spending sufficient time on their academic tasks. ${ }^{46}$ Earlier studies suggested that society perceives individuals who spend much time on sports and do not perform well academically through the use of the term "jock," ${ }^{47}$ which could be a potential explanation for negative associations. ${ }^{17}$ However, results from systematic reviews that investigate the associations with academic performance and PA refute this notion. ${ }^{11,12,21,48}$ More research is necessary that investigates the environments where students take part in daily MVPA and have below average and average PSP.
Roughly $5 \%$ of young adolescents perceived their PSP as below average. Despite representing a small proportion of children, the association with MVPA was the lowest. Among boys in France and Slovenia, there were negative associations between young adolescents who were physically active and had average or good PSP. This could have implications on the perceptions of sports athletes and their low PSP. ${ }^{17}$ Perceptions of time spent on training in sports instead of improving school performance may be common among young adolescents in these countries. For example, Slovenia has nationalized biannual health-related fitness assessments, and this has been praised for enhancing the prevalence of active adolescents. ${ }^{49}$ The link between national fitness testing and overall academic performance would be an important area to investigate, as these tests are linked with the national education department to be part of overall assessments. ${ }^{50}$ At the individual level, the notion of mastery as motivation to be physically active may take precedence in other aspects in life, such as the importance of good PSP. ${ }^{51}$ Moreover, there are more sporting competition opportunities as individuals get older, ${ }^{10}$ and these may serve as another form of motivation for young adolescents to spend more time on PA rather than focus on their perceptions of their own school performance.

At the other end of the PSP spectrum (very good), 5 to 6 days of MVPA was significantly associated in 33 countries, and daily MVPA, in half of the countries. The results of our study further indicate that the associations are nonlinear and that the doseresponse relationships are more complex as children get older. For example, Badura et $\mathrm{al}^{52}$ created clusters of leisure-time activities, whereby all-rounders (had sport and nonsport hobbies) had the strongest positive association with PSP, indicating that a balance of time is important over 7 days of MVPA. In addition, physical fitness has been consistently associated with academic performance of young adolescents in various longitudinal studies..$^{23,53}$ Furthermore, the positive effects of PA on social skills and social networks, ${ }^{12}$ and stress regulation ${ }^{54}$ are discussed as mediating factors to explain the link between PA and educational outcomes. Nonetheless, it should be noted that there were 7 countries (Armenia, Austria, Greenland, Republic of Moldova, Malta, the Netherlands, and Ukraine) without significant associations between PSP and MVPA for all ages and both sexes. Further in-depth studies are necessary to explain these country-specific exceptions.

The majority of studies conducted on the relationships between academic performance and PA have used school grades or results from cognitive tests. ${ }^{21}$ There is a lack of investigations on the perception of academic pressure in understanding the relationships with PA. Children's perception of their academic ability is an important indicator of actual progress. It is important to consider the voice of the children in the decision-making processes related to children. ${ }^{55}$ The HBSC study has been successful in delivering this message by involving children in the research process as research participants. Another strength in the design of HBSC is the inclusion of measures that are comparable across different countries and are uniformly applicable to young adolescents aged 11 to 15 years. ${ }^{56}$ On the other hand, there are differences among education systems in various parts of the world. Academic achievement is a fundamental desired outcome of a school system. The usual indicators of academic achievement are school grades because they are supposed to reflect how well a student has mastered specific learning standards. Many factors, such as idiosyncratic classroom norms for performance and investment in schoolwork or classroom climate, may influence students' perceptions of their performance. ${ }^{13}$ Despite this limitation to the single item on PSP, the responses do seem to reflect actual academic grades across different educational systems and countries. ${ }^{25}$ 
Some limitations of this study are important to consider when interpreting the results. The study was based on cross-sectional data from an international survey carried out in 2013-14. Therefore, assumptions based on causality have not been tested nor reported, and future large-scale longitudinal studies including potential mediators and moderators are required. Another limitation is the nature of the data collection. Countries within the HBSC network were responsible for their own data collection through an international protocol. At the time of the data collection, anonymous survey responses do not allow for linking with actual school report grades, and thus, may bring sampling bias to the study. As such, measures of self-reporting of PA and academic performance were used, despite the risk of some recall errors. Moreover, the data were pooled and larger population sample sizes allow for smaller effects, and adjustments were not made based on the sensitivity of selfreflection changing with age. In addition, to reduce the complexity of combined and individual country data, other uncontrolled confounders, for example, other family members and parental education, were not included in the models.

\section{Conclusions}

As young adolescents age from 11 to 15 years, there is a reduction in MVPA and PSP that becomes increasingly significant. The results of this study support the notion that any improvement in PA from inactivity was associated with increased PSP, with the optimal state being between 5 and 6 days. There is an indication that performing well at school involves a balancing act with the amount of PA a young adolescent engages in; accordingly, an optimal frequency of 5 to 6 days was noted across sex and age groups after controlling for FAS3. The patterns remain largely similar across the included countries; therefore, important information for public health should be noted from these results when optimizing PSP and physical activities. Young adolescents' perceptions of school performance and PA are an important attribute to account for when planning public health and health promotion strategies. This is especially true when health promotion strategies should be integrated into the educational system, as PA promotion must be considered with a maximization of educational outcomes.

\section{Acknowledgments}

This work received support from European Regional Development FundProject "Effective Use of Social Research Studies for Practice" (No. CZ.02.1.01/0.0/0.0/16_025/0007294). This work was also funded by the Wallonia-Brussels Federation, the Birth and Children Office (ONE), the Walloon Region, and the French Community Commission. Researchers performed the study (study design, data collection, analyses, interpretation and writing) independently of the funding bodies. All authors declare no conflict of interest exists. All procedures performed in studies involving human participants were in accordance with the ethical standards of the institutional and/or national research committee and with the 1964 Helsinki Declaration and its later amendments or comparable ethical standards. Informed consent was obtained from all individual participants included in the study. The data used from this study can be requested from the HBSC Data Centre dmc@hbsc.org.

\section{References}

1. 2018 Physical Activity Guidelines Advisory Committee. 2018 Physical Activity Guidelines Advisory Committee Scientific Report. 2018.
2. Rodriguez-Ayllon M, Cadenas-Sánchez C, Estévez-López F, et al. Role of physical activity and sedentary behavior in the mental health of preschoolers, children and adolescents: a systematic review and meta-analysis. Sports Med. 2019;49(9):1383-1410. PubMed ID: 30993594 doi:10.1007/s40279-019-01099-5

3. World Health Organization. Global Recommendations on Physical Activity for Health. Geneva, Switzerland: World Health Organization. 2010.

4. Parrish A, Tremblay MS, Carson S, et al. Comparing and assessing physical activity guidelines for children and adolescents: a systematic literature review and analysis. Int J Behav Nutr Phys Act. 2020; 17(1):16. PubMed ID: 32041635 doi:10.1186/s12966-020-0914-2

5. Guthold R, Stevens GA, Riley LM, Bull FC. Global trends in insufficient physical activity among adolescents: a pooled analysis of 298 population-based surveys with 1.6 million participants. Lancet Child Adolesc Health. 2020;4(1):23-35. PubMed ID: 31761562 doi:10.1016/S2352-4642(19)30323-2

6. Inchley J, Currie D, Young T, et al. Growing up unequal: gender and socioeconomic differences in young people's health and well-being. Health Behaviour in School-aged Children (HBSC) study: International report from the 2013/2014 survey. Health Policy for Children and Adolescents, No. 7. 2016.

7. Biddle SJH, Atkin AJ, Cavill N, Foster C. Correlates of physical activity in youth: a review of quantitative systematic reviews. Int Rev Sport Exerc Psychol. 2011;4(1):25-49. doi:10.1080/1750984X. 2010.548528

8. Sawyer SM, Azzopardi PS, Wickremarathne D, Patton GC. The age of adolescence. Lancet Child Adolesc Health. 2018;2(3):223-228. PubMed ID: 30169257 doi:10.1016/S23524642(18)30022-1

9. Klinger DA, Freeman JG, Bilz L, et al. Cross-national trends in perceived school pressure by gender and age from 1994 to 2010. Eur J Public Health. 2015;25(Suppl 2):51-56. doi:10.1093/eurpub/ ckv027

10. Directorate-General for Education and Culture. EU Guidelines on Dual Careers of Athletes; Recommended Policy Actions in Support of Dual Careers in High-Performance Sport. Brussels, Belgium: European Commission; 2012.

11. Singh A, Uijtdewillign L, Twisk JWR, Mechelen WV, Chinapaw MJ. Physical activity and performance at school: a systematic review of the literature including a methodological quality assessment. Arch Pediatr Adolesc Med. 2012;166(1):49-55. PubMed ID: 22213750 doi:10.1001/archpediatrics.2011.716

12. Busch V, Loyen A, Lodder M, Augustinus JPS, van Yperen TA, de Leeuw JRJ. The effects of adolescent health-related behavior on academic performance: a systematic review of the longitudinal evidence. Rev Educ Res. 2014;84(2):245-274. doi:10.3102/003465 4313518441

13. Marques A, Santos DA, Hillman CH, Sardinha LB. How does academic achievement relate to cardiorespiratory fitness, self-reported physical activity and objectively reported physical activity: a systematic review in children and adolescents aged 6-18 years. Br J Sports Med. 2018; 52(16):1039. doi:10.1136/bjsports-2016-097361

14. Harter S. The Construction of the Self: A Developmental Perspective. New York, NY: The Guilford Press; 1999:413.

15. Lintunen T. Development of self-perceptions during the school years. In: Auweele YV, Bakker F, Biddle SJH, Durand M, Seiler R, eds. Psychology for Physical Educators. 1st ed. Champaign, IL: Human Kinetics; 1999:115-134.

16. Esnaola I, Sesé A, Antonio-Agirre I, Azpiazu L. The development of multiple self-concept dimensions during adolescence. J Res Adolesc. 2020;30:100-114. PubMed ID: 30156745 doi:10.1111/jora.12451 
17. Koivusilta LK, Rimpelä AH, Rimpelä M, Vikat A. Health behaviorbased selection into educational tracks starts in early adolescence. Health Educ Res. 2001;16(2):201-214. PubMed ID: 11345662 doi:10.1093/her/16.2.201

18. LeBlanc MM, Martin CK, Han H, et al. Adiposity and physical activity are not related to academic achievement in school-aged children. J Dev Behav Pediatr. 2012;33(6):486-494. PubMed ID: 22617499 doi:10.1097/DBP.0b013e31825b849e

19. Maher C, Lewis L, Katzmarzyk PT, Dumuid D, Cassidy L, Olds T. The associations between physical activity, sedentary behaviour and academic performance. J Sci Med Sport. 2016;19(12):1004-1009. PubMed ID: 26971300 doi:10.1016/j.jsams.2016.02.010

20. Barth Vedøy I, Anderssen SA, Tjomsland HE, Skulberg KR, Thurston M. Physical activity, mental health and academic achievement: a cross-sectional study of Norwegian adolescents. Ment Health Phys Act. 2020;18:100322. doi:10.1016/j.mhpa.2020.100322

21. Suhrcke M, de Paz Nieves C. The Impact of Health and Health Behaviours on Educational Outcomes in High Income Countries: A Review of the Evidence. Copenhagen, Denmark: WHO Regional Office for Europe; 2011.

22. Howie EK, Pate RR. Physical activity and academic achievement in children: a historical perspective. J Sport Health Sci. 2012;1(3):160169. doi:10.1016/j.jshs.2012.09.003

23. Donnelly J, Hillman CH, Castelli DM, et al. Physical activity, fitness, cognitive function, and academic achievement in children: a systematic review. Med Sci Sports Exerc. 2016;48(6): 1197-1222. PubMed ID: 27182986 doi:10.1249/MSS.0000000 000000901

24. Carlson JJ, Eisenmann JC, Pfeiffer KA, et al. (S)partners for heart health: a school-based program for enhancing physical activity and nutrition to promote cardiovascular health in 5(th) grade students. BMC Public Health. 2008;8(1):420. PubMed ID: 19102777 doi:10 .1186/1471-2458-8-420

25. Felder-Puig R, Griebler R, Samdal O, King MA, Freeman JG, Duer W. Does the school performance variable used in the international Health Behavior in School-aged Children (HBSC) study reflect students' school grades? J Sch Health. 2012;82(9):404-409. PubMed ID: 22882103

26. Stringer RW, Heath N. Academic self-perception and its relationship to academic performance. Can J Educ. 2008;31(2):327-345.

27. Geiger MA, Cooper EA. Predicting academic performance: the impact of expectancy and needs theory. J Exp Educ. 1995;63(3): 251-262. doi:10.1080/00220973.1995.9943812

28. Taylor G, Jungert T, Mageau GA, et al. A self-determination theory approach to predicting school achievement over time: the unique role of intrinsic motivation. Contemp Educ Psychol. 2014;39(4):342-358. doi:10.1016/j.cedpsych.2014.08.002

29. Hagborg WJ. The Rosenberg self-esteem scale and Harter's selfperception profile for adolescents: a concurrent validity study. Psychol Sch. 1993;30(2):132-136. doi:10.1002/1520-6807(199304)30: 2<132::AID-PITS2310300205>3.0.CO;2-Z

30. Brewer BW, Van Raalte JL, Linder DE. Athletic identity: Hercules' muscles or Achilles heel? Int J Sport Psychol. 1993;24: 237-254.

31. Martin G, Richardson AS, Bergen HA, Roeger L, Allison S. Perceived academic performance, self-esteem and locus of control as indicators of need for assessment of adolescent suicide risk: implications for teachers. J Adoles Health. 2005;28(1):75-87. doi:10.1016/j.adolescence.2004.04.005

32. Currie C, Inchley J, Molcho M, Lenzi M, Veselska Z, Wild F. Health Behaviour in School-Aged Children (HBSC) Study Protocol: Background, Methodology and Mandatory Items for the 2013/14
Survey. St. Andrews, Scotland: Child and Adolescent Health Research Unit (CAHRU); 2014.

33. Vereecken C. Paper pencil versus pc administered querying of a study on health behaviour in school-aged children. Arch Public Health. 2001;59(1):43-61.

34. Liu Y, Wang M, Villberg J, et al. Reliability and validity of Family Affluence Scale (FAS II) among adolescents in Beijing, China. Child Indic Res. 2012;5(2):235-251. doi:10.1007/s12187-011-9131-5

35. Hardie Murphy M, Rowe DA, Belton S, Woods CB. Validity of a twoitem physical activity questionnaire for assessing attainment of physical activity guidelines in youth. BMC Public Health. 2015;15(1):1080. PubMed ID: 26498827 doi:10.1186/s12889-015-2418-6

36. Ng KW, Hämylä R, Tynjälä J, et al. Test-retest reliability of adolescents' self-reported physical activity item in two consecutive surveys. Arch Public Health. 2019;77(1):9. doi:10.1186/s13690-019-0335-3

37. Biddle SJH, Gorely T, Pearson N, Bull FC. An assessment of selfreported physical activity instruments in young people for population surveillance: project ALPHA. Int J Behav Nutr Phys Act. 2011;8(1):1. doi:10.1186/1479-5868-8-1

38. Torsheim T, Cavallo F, Levin KA, et al. Psychometric validation of the revised family affluence scale: a latent variable approach. Child Indic Res. 2016;9(3):771-784. PubMed ID: 27489572 doi:10.1007/ s12187-015-9339-x

39. Merlo J, Chaix B, Ohlsson H, et al. A brief conceptual tutorial of multilevel analysis in social epidemiology: using measures of clustering in multilevel logistic regression to investigate contextual phenomena. J Epidemiol Community Health. 2006;60(4):290-297. PubMed ID: 16537344 doi:10.1136/jech.2004.029454

40. Shieh G. Choosing the best index for the average score intraclass correlation coefficient. Behav Res Methods. 2016;48(3):994-1003. PubMed ID: 26182855 doi:10.3758/s13428-015-0623-y

41. Hox J. Multilevel Analysis: Techniques and Applications. Mahwah, NJ: Lawrence Erlbaum Associates; 2002.

42. Boyce WF, Torsheim T, Currie C, Zambon A. The family affluence scale as a measure of national wealth: validation of an adolescent selfreport measure. Soc Indic Res. 2006;78(3):473-487. doi:10.1007/ s11205-005-1607-6

43. Meeusen R, Schaefer S, Tomporowski PD, Bailey R. Physical Activity and Educational Achievement: Insights from Exercise Neuroscience. Abingdon, Oxon: Routledge; 2018.

44. Marcenaro-Gutierrez O, Lopez-Agudo LA, Ropero-García MA. Gender differences in adolescents' academic achievement. YOUNG. 2018;26(3):250-270. doi:10.1177/1103308817715163

45. Kalman M, Inchley J, Sigmundova D, et al. Secular trends in moderate-to-vigorous physical activity in 32 countries from 2002 to 2010: a cross-national perspective. Eur J Public Health. 2015;25(Suppl 2):37-40. doi:10.1093/eurpub/ckv024

46. Ryba TV, Aunola K, Kalaja S, Selänne H, Ronkainen NJ, Nurmi J. A new perspective on adolescent athletes' transition into upper secondary school: a longitudinal mixed methods study protocol. Cogent Psychol. 2016;3(1):1142412. doi:10.1080/23311908.2016.1142412

47. Nelson ES. How the myth of the dumb jock becomes fact: a developmental view for counselors. Couns Values. 1983;27(3): 176-185. doi:10.1002/j.2161-007X.1983.tb01131.x

48. Centers for Disease Control and Prevention. The Association Between School Based Physical Activity, Including Physical Education and Academic Performance. Atlanta, GA: U.S. Department of Health and Human Services; 2010. www.cdc.gov/HealthyYouth

49. Tremblay MS, Barnes JD, González SA, et al. Global matrix 2.0: report card grades on the physical activity of children and youth comparing 38 countries. J Phys Act Health. 2016;13(11 Suppl 2): S343-S366. doi:10.1123/jpah.2016-0594 
50. Shephard RJ. Teaching health and fitness to the growing child: the physical educator. In: Shephard RJ, ed. A History of Health and Fitness: Implications for Policy Today. New York, NY: Springer; 2018:285-311. doi:10.1007/978-3-319-65097-5

51. Diseth $\AA$, Samdal O. Autonomy support and achievement goals as predictors of perceived school performance and life satisfaction in the transition between lower and upper secondary school. Soc Psychol Educ. 2014;17(2):269-291. doi:10.1007/s11218-013-9244-4

52. Badura P, Sigmund E, Madarasova Geckova A, et al. Is participation in organized leisure-time activities associated with school performance in adolescence? PLoS One. 2016;11(4):e0153276. PubMed ID: 27073841 doi:10.1371/journal.pone.0153276

53. London RA, Sebastian C. A longitudinal examination of the link between youth physical fitness and academic achievement. J Sch
Health. 2011;81(7):400-408. PubMed ID: 21668880 doi:10.1111/j. 1746-1561.2011.00608.x

54. Taras H. Physical activity and student performance at school. J Sch Health. 2005;75(6):214-218. PubMed ID: 16014127 doi:10.1111/j. 1746-1561.2005.00026.x

55. Andersen CS, Dolva A. Children's perspective on their right to participate in decision-making according to the united nations convention on the rights of the child article 12 [published online ahead of print May 27, 2014]. Phys Occup Ther Pediatr. doi:10.3109/ 01942638.2014.918075

56. Currie C, Alemán-Díaz AY. Building knowledge on adolescent health: reflections on the contribution of the Health Behaviour in School-aged Children (HBSC) study. Eur J Public Health. 2015;25(Suppl 2):4-6. doi:10.1093/eurpub/ckv017 